\title{
Evaluating the Impact of Routing on QoS of VoIP over MANET Wireless Networks
}

\author{
Azeddien M. Sllame \\ Faculty of Information Technology, University of Tripoli, Tripoli, Libya \\ Email: Aziz239@yahoo.com, a.sllame@uot.edu.ly
}

How to cite this paper: Sllame, A.M. (2017) Evaluating the Impact of Routing on QoS of VoIP over MANET Wireless Networks. Open Access Library Journal, 4: e3361.

https://doi.org/10.4236/oalib.1103361

Received: January 9, 2017

Accepted: February 17, 2017

Published: February 20, 2017

Copyright $\odot 2017$ by author and Open Access Library Inc.

This work is licensed under the Creative Commons Attribution International License (CC BY 4.0).

http://creativecommons.org/licenses/by/4.0/

\begin{abstract}
In this paper, a performance analysis study for VoIP over mobile wireless ad hoc networks (MANET) is carried out using OPNET tool. The AODV, DSR, TORA, OLSR, and GRP routing protocols have been used as candidates to evaluate the impact of routing on quality of service (QoS) of VoIP application over MANET networks. Three different queuing mechanisms (FIFO, PQ, and WFQ) have been used also as nominee of queuing techniques. The VoIP is applied and the QoS is measured in terms of jitter delay, end-to-end packet delay, and wireless LAN media access delay and wireless throughput of 802.11 g technology with 54 Mbps.
\end{abstract}

\section{Subject Areas}

Network Modeling and Simulation

\section{Keywords}

MANET, Ad Hoc Wireless Networks, Routing Protocol, VoIP, Queuing, OPNET, Modeling, Simulation

\section{Introduction}

At the present time, computer networks are one of the most important assets in cloud computing era where networks are used to distribute, store, and share the information across the world by users, companies, governmental institutions, scientific communities, and social networks. Moreover, in every moment there are hundred-millions of data files, multimedia streaming videos, VoIP calls, and emails are transferred between users all over the world by using the Internet. Multimedia transmission needs specified end-to-end delay limits, very low jitter and an efficient routing with an acceptable quality of service (QoS) level, which makes routing as one of the critical concerns while implementing computer 
networks across the world, especially when deploying wireless technology such as mobile networks. A mobile wireless ad hoc network (MANET) is a set of wireless independent nodes dynamically founding a temporary network by means of wireless links in an infrastructure less structure with decentralized management. All the nodes in the ad hoc networks form together a wireless asymmetrical communication links using minimum organization and control attempts. Mobility of the nodes makes the changeable topology of the MANET in which the routing situation is changing every moment by the movement of different nodes. Thus making the data transmission between nodes takes different hops each time the movement occurs where each node contributes in the routing between nodes in a store and forward fashion [1] [2].

Ad hoc networks can be implemented by different wireless technologies such as Bluetooth, IEEE 802.11, and Ultra-Wide Band (UWB) enabling established ad hoc wireless networks to benefit from different capabilities of such networking models to realize different wireless networks according to the requirements and employed situation. In addition, ad hoc networks can be set up rapidly and nodes can be added or removed logically or physically according to the network's requirements. However, when any link failure happens due to the node failure or leaving situation, all the affected nodes will initiate requests to establish new routes to reconnect up with all unreachable nodes and to reconstruct the connectivity between the existing operational nodes of the ad hoc wireless network to preserve the operational network. Therefore, this makes routing process a critical concern in ad hoc networks whereas in ad hoc networks the nodes perform the routing by forwarding packets through intermediate nodes from sender to the destination, since the nodes in the wireless ad hoc networks are operating as a host and as a router. So it is used in disaster rescue, scientific conferences, and military operations. The wireless ad hoc networks have the following advantages: it is easy to build network with decentralized flexible architecture and has no infrastructure, and can be constructed with lower cost devices, mobility which is a good feature for quick deployment. On the other hand, wireless ad hoc networks have the following disadvantages: higher error bit-rate than wire networks, multi-hop routing so packets may pass through multiple links of mobile nodes to reach the destination; dynamic topology makes a scalability problem to the ad hoc networks; security problems due to decentralized infrastructure less organization, diverse nodes' capabilities with different linking techniques; and node limited resources such as battery power and bandwidth of the node [1] [3].

Performance evaluation of different components of the networks has become a significant issue in recent years due to the widespread using of Internet in different daily life aspect such as in multimedia networking, social networking, e-commerce, and e-learning. Routing is one of the most networking components that affect the packet's transmission, hence it is considered in many research papers for evaluation in order to improve and design new routing protocols for future use with huge data transfer around the world in the cloud computing era. Typical performance measures (metrics) include system/network-oriented mea- 
sures such as throughput, end-to-end delay, jitter, packet losses, and applicationoriented measures such as number of calls per hour, signal-to-noise ratio, rate of calls injection procedure (serial, random, concurrent) for VoIP application; as an example [4].

However, the performance of computer networking systems can be evaluated by measurement/real analysis, systematic modeling, and simulation techniques. Modeling and simulation is used in computer networking field to support the design knowledge and to enhance building more hands-on skills to the students and designers in order to fine-prepare them for their future profession. In computer networking specialization, modeling and simulations using software tools are playing a critical role in teaching, designing, and evaluating networks because prototyping using real networks' devices are too expensive and usually networks designed to be run over several parts or many buildings in a city or a country. Moreover, current networking design practice combines different wire and wireless networking technologies which have distinct and complex protocols, multiple devices, and variety of communication standards making the modeling and simulation is the first choice for computer network educational/evaluation design courses [5]. Therefore, modeling and simulation tools are put in effect to support prototyping and experimenting to overcome the cost and space restrictions in implementing real computer networking projects which in turn enrich designers' participation in open educational environments and design activities. There are a range of simulation tools like OPNET, NS-2, NetSim, packet tracer, GNS3, Open Sim MPLS, and OMNet++ that are accessible for the purpose of modeling and simulation at different levels of the computer networks. However, the selection of a simulator is subject to the simulator's features and the requirements of the application under design. OPNET is one of the simulation tools that can be used to analyze, investigate, and design computer networks [4] [5] [6].

The main objective of this research paper is to verify whether the routing protocols affect VoIP end-to-end delay, and delay jitter in a MANET using a set of routing protocols which apply distinct route calculation techniques to find a route between a sender and destination pair.

This paper is organized as follows: related work is outlined in Section 2. The routing in MANET network is briefly discussed in Section 3. Section 4 introduces the reader to the QoS metrics and queuing. The case study example and corresponding results are described in Section 5. Section 6 outlines the comparison results between selected wireless ad hoc networks routing protocols. Finally, conclusion remarks are given in Section 7.

\section{Related Work}

Many research papers on the paper's topic are reported in literature. L. Audah et al. in [7] had described a VoIP evaluation using different codecs techniques using NS-2 simulation tool with multiple traffic connections transferred over the Internet. The performance was measured in terms of end-to-end delay, packet's 
loss ratio, throughput, and delay jitter. Sandeep Ravikanti and Gadasu Preeti in [8] evaluated VoIP using Dynamic Source Routing (DSR), Ad hoc On-demand Distance Vector (AODV), Temporally-Ordered Routing Algorithm (TORA) using OPNET simulation tool. However, their research resulted in the suitability of TORA protocol for VoIP application; but there are no details about the quantity of the VoIP traffic generated in their simulation study. In [9] Hetal Jasani conducted a QoS study for VoIP application using AODV and DSR routing protocols using OPNET tool. The simulation was run with IEEE 802.11n for 30 nodes in a region of $2 \mathrm{~km} \times 2 \mathrm{~km}$ area with 5 minutes simulation time interval; which is very short simulation period. Their results showed that AODV is suitable for VoIP application in MANET networks. Ramon Sanchez-Iborra, Maria-Dolores Cano had described in [10] an approach to a cross layer-based quality of experience improvement for MANET routing protocols in which they presented a comparison among OLSR, AODV, and BATMAN for video transmission with IEEE $802.11 \mathrm{~g}$ at $54 \mathrm{Mbps}$ using Omnet++ v4.4 simulation environment. In [11] Lin Cai et al. surveyed the latest improvements of VoIP over WLAN in terms of capacity analysis, call admission provisions, and medium access control (MAC) layer QoS enhancement devices. M. Vijayakumar1 and V. Karthikeyani in [12] described an enhanced perceptive queuing technique (CBCRTQ) for QoS of VoIP over MANET network using AODV routing protocol with NS-2 simulation tool. In [13] Shiraz Mohammed et al. evaluated VoIP over MANET using OLSR and TORA with OPNET simulation tool. However, the results showed that OLSR routing protocol is a good candidate for VoIP application. Gianni A. Di Caro et al. [14] described a simulation study of routing performance of AODV and Ant Hoc Net routing protocols for VoIP over MANET network using QualNet simulator in a realistic urban environment. Khushboo Mittal and Preeti Sharma in [15] compared QoS of VoIP application over MANET using three routing protocols i.e. OLSR, AODV, and TORA by means of OPNET simulator tool. Said El brak in [16] described a QoS performance study of VoIP over MANET with different audio codecs employing AODV, OLSR routing protocol using NS-2 simulator. The study showed that how codecs affect the QoS of the VoIP with varying hops count number.

\section{Routing in MANET Networks}

In MANET, a pair of nodes exchanges packets over a direct wireless node, or over a chain of wireless nodes. However, for MANET efficiency the employed routing protocol needs to deal with many MANET networks limitations, such as battery lifetime, transmission errors, channel capacity, and rapidly changing topology due to node's mobility. Moreover, Node density which represents the number of nodes existing in the MANET network is playing an important role in the routing, since some routing protocols; such as OLSR; is producing better results when applied with dense MANET networks than in the less-dense MANET networks. In this section a brief description of all routing protocols that are used in this paper work is given below [1]. 


\subsection{Proactive Routing Protocols}

This kind of routing protocols is continuously evaluating the routes between active nodes and keeps a topology table that covers the active nodes connectivity. The table is continuously retained up to date, which give the proactive protocols the name of table-driven. Therefore, all active nodes exchange routing information gathered in such tables with neighbors to maintain an accurate scene of the current ad hoc network topology. However, proactive routing protocols attempt to maintain reliable, up-to-date routing information when a route is needed; i.e., one route may be arranged immediately. Whenever the network topology changes proactive routing protocols react by disseminating updates throughout the network to preserve a consistent view. Thus, this will consume the bandwidth and may keep up routes that may never be used. Proactive routing has high routing overhead than reactive routing protocols.

Examples of proactive (table-driven) are Destination Sequenced Distance Vector routing (DSDV), Cluster-head Gateway Switch routing (CGSR), and Wireless Routing Protocol (WRP), Geographic routing protocol (GRP), and Optimized Link State Routing (OLSR) protocol.

\subsection{Reactive Routing Protocols}

This class also known as on-demand routing protocols since the node requests the route only when there is data to be sent by the node. In reactive routing the source node initiates the route discovery method to find a route to the destination by using some global search technique. This will introduce a delay on the data transfer. Reactive routing is suitable for highly dynamic networks since no routing arrangements created a priori. However, because of employing high flood-search when a transfer is needed between certain nodes with mobility and distributed traffic over the MANET network-an overhead will be introduced.

Examples of reactive (on-demand) are Ad hoc On-Demand Distance Vector (AODV), Dynamic Source Routing (DSR), and Temporary Ordered Routing Algorithm (TORA).

\subsection{Hybrid Routing Protocol}

Hybrid protocols are the combinations of reactive and proactive protocols and inherit advantages of these two types of protocols. Hybrid routing protocols possibly will demonstrate proactive or reactive behaviors depending on the circumstances, therefore will deliver flexibility to the wireless network.

However, with reactive protocols there is no guarantee to find the shortest path since after discovering a path no effort is made to find a better path until the current path becomes unusable due to mobility of the network nodes. Meanwhile, for proactive protocols there is a possibility to have shortest paths because routes are continuously updated and kept in nodes' routing tables.

\subsection{The Ad-Hoc on Demand Distance Vector (AODV) Protocol}

AODV is a reactive on demand distance-vector routing protocol. AODV is an 
enhancement of the DSDV routing protocol. Unlike DSDV the AODV protocol reduces the number of broadcasts and works well with highly active networks. AODV as a reactive protocol searches routes only when data need to be transferred between MANET nodes. However, such routes are kept in nodes' routing tables and route entries at nodes routing tables are expired if not used for a specified time interval. Therefore, this mechanism reduces the size of the route cache inside the memory of the nodes. AODV reduces the number of broadcasted routing messages in the network by issuing link failures notifications only to affected nodes as well as the AODV owns the loop freedom feature [1].

\subsection{Dynamic Source Routing Protocol (DSR)}

DSR is a reactive, source-routed routing protocol particularly originated for dynamic networks. However, in DSR every node reserves all required information of all routing routes from this node to all other nodes in the topology. Essentially, the source node in DSR routing initiates the "Route Discovery" technique to discover a routing path to any targeted node whenever there is no record about that node in the node's routing database. Thus, the discovered route information is then stored temporarily in the nodes route cache. In addition, DSR applies "Route Maintenance" procedure to keep the routes at any time there is a link missing in the routes or whenever any topology alterations occur. Therefore, DSR reconstructs failed routes quickly to facilitate the node accessibility by other nodes. Hence, once a route is set up, the sending node transfers packets with headers saving all the path information details on the way to the receiving end. In addition, individual node through that path advances packets to the following node in the topology conforming to control information enclosed in the header of the packet. DSR keeps routes of designated nodes only; thus; DSR does not make any route advertisements with untargeted nodes. On another hand, DSR is designed to be also a source routing protocol which makes transmission of periodic hello messages by the node to inform other nodes about its presence in the topology unnecessary. Therefore, the intermediate nodes of that topology do not need to maintain the route information of that initiated source routing between the source and destination pair [1].

\subsection{Temporally-Ordered Routing Algorithm (TORA)}

TORA is a reactive, adaptive distributed routing protocol developed to be employed in dynamic multihop networks. TORA is founded on link reversal algorithm; it maintains a directed acyclic graph (DAG); rooted at the destination; to generate multiple routing paths according to the source to sender requests. TORA is intended to diminish replies to topology's changes. The important characteristic of the TORA is its response to link failures. Then, TORA erases invalid routes, explores new routes, and constructs new routes in a single-pass of the distributed algorithm. Hence, in TORA the control messages are usually localized to a small group of nodes; thus ensuring that all routes are loop-free and provides several paths for any two connected nodes [1]. 


\subsection{The Optimized Link State Routing (OLSR) Protocol}

OLSR is a table-driven proactive routing protocol, essentially it is a link-state routing protocol optimized for use in MANET networks. OLSR protocol repeatedly exchanges the network topology's information with other nodes in the network. The OLSR carry out hop-by-hop routing. OLSR protocol employs the multipoint relays (MPR) technique in order to reduce the number of neighbors that are intended to receive the broadcasting of the control traffic messages by selecting a group of routers that will receive to forward the path searching process flooding messages. Hence, every router (node) in the way to destination will select subset of its neighbors that are targeted to receive the broadcast message. OLSR protocol is an appropriate selection for large and dense networks, but it might not find the shortest path due to the MPR mechanism [1].

\subsection{Geographic Routing Protocol (GRP)}

GRP is a proactive routing protocol and is developed to overcome the drawbacks of the topology based routing protocols. Also known as position based routing. GRP protocol has grown in its use in MANET networks as a result of its scalability feature. In GRP the routing is based on the shortest geographical distance between source and destination. However, the source node at all times finds the nearest neighbor node to the destination and sends the packet to it while waiting for the packet to reach its destination. Thus, every node utilizes the geographical position system (GPS) to detect and recognize its particular position. Basically, GRP initiates flooding to the entire network in order to identify and learn about all nodes in the network's topology. Hence, after finishing the initial flooding phase, each node in the network will be aware of the initial position of all other reachable nodes in the whole network. The GRP uses Hello protocol and updates nodes' positions based on the distance moved and the crossed neighbors during the mobility. However, the implementation of the GRP on the OPNET includes backtracking feature in such a way that packets on blocked routes are backtracked to previous nodes to facilitate the rerouting of such packets along another valid paths [1] [6].

\section{Quality of Service}

The quality of service (QoS) is a very important issue in transmitting multimedia over networks and the network that offers QoS is the network which delivers the transmitted data with a definite quality assurance level between senders and receivers within the imposed delivery time limits for real-time applications. When working with multimedia we will meet three essential restrictions; first multimedia streams typically need a considerable higher bandwidth than any ordinary data streams. Second, most of the multimedia applications are of real-time traffic type such as VoIP [17] [18]. Thus, they need to be played back at the same order that had sent from the source. Third, multimedia flow streams are commonly composed of huge data in bulky blocks. Therefore, working with multimedia data over Internet is a challenge that requires dealing with multicast routing, 
higher bandwidth in network interconnecting nodes, use protocols that are developed to deal with real-time multimedia streaming such as real-time transmission protocol (RTP).

In practice, QoS is defined as the set of methods that are used to regulate the network packet delay jitter, packet end-to-end delay, and packet loss to achieve applicable throughput to satisfy the requirements of the real-time applications from bandwidth and packet transfer delay.

- The delay jitter is defined as the delay variation between two successive packets in the same traffic flow. However, queuing, rerouting, and added processing delays are the main reasons of the delay jitter.

- The end-to-end delay is defined as the time needed to send a packet from the source until it reaches the final destination.

- A packet loss is defined as the number of lost packets that is happened inside the network at definite time interval during the packet transfer process from the sender to the receiver.

As the usage of the multimedia streaming has been grown with different applications over the Internet the QoS is enforced to be included into networking infrastructure. This introduced new functions such as packet classification and queuing in addition to scheduling inside the router's architecture. Packet classification is the function of differentiating packets into a number of classes with the aim of operating necessary actions or definite rules to each class of data streams such as VoIP or real-time video streaming in order to enhance QoS of the network. Yet, to do packet classification the router will possibly need to check other packet fields such as destination address or payload type. After classification, other traffic treatment procedures like policing and marking can be applied [17] [18].

\section{Queuing}

Queuing is the function of storing packets temporarily inside the router's queues before getting transmitted toward their final destination. However, the packet's transmission is influenced by numerous issues such as the packet's service class or the level of service assurance associated with the packet's class. Therefore, different queues have different priority levels are employed and every queue is assigned a particular amount of the bandwidth on the same output link according to their priority value. Scheduling technique is used to select the next packet to be transferred from such queues according to the prearranged bandwidth for each queue. Normally, the buffering space is guaranteed for the traffic that is belonging to the real-time classes, whereas discarding policies may be applied to the normal traffic classes. Scheduling possibly will reorder the traffic flows regarding to their arrival times. Scheduling is always used with queuing to fit in the required stream's bandwidth while keeping queueing delays at the preferred values for the given application. Scheduling determines the time order when different traffic flows are forwarded through the current router (the MANET node). In this paper we are using three kinds of queuing [2] [18]. 
First class is First In First Out (FIFO) queuing in which all packets is treated equally. Therefore bursty sources can occupy the bandwidth and cause delays in real-time streams which can experience dropping to real-time traffic since the less important traffic occupies the queue. Thus, FIFO does not support any QoS guarantee [18].

Second class is priorityqueuing $(\mathrm{PQ})$ packets are classified to a definite priority class. However, those streams that are belonging to the higher priority class are sent before all lower priority traffic since the higher priority class traffic guaranteed their delivery in timing and bandwidth. PQ is a traffic differentiation technique, but it is not optimal, since it disturbs handling of low priority queue's packets which may get discarded first when the congestion happens. PQ queuing isn't a max-min fair method, so it is need to be combined with any other mechanism to control traffic into queues [18].

Third class is Weighted Fair Queuing (WFQ). Fair queuing technique is designed to overcome the limitations of the FIFO and PQ queuing. The fair queuing classifies the packets into several groups; each group has a particular queue and each queue has a predetermined buffer size. In WFQ each queue is given a weight which equal to its share of the link. Thus, this prevents the larger flows from consuming network's bandwidth since WFQ employs sorting and interleaving of individual packets by flow and then queue each flow based on the volume of traffic in this flow. This makes WFQ involving heavy computational overhead per packet in the flows which increases complexity to WFQ realization inside the routers. Consequently, WFQ is a max-min fair technique and it provides some QoS to real-time streams [18].

\section{The Case Study Example and Results}

The simulations consist of twelve scenarios with considering the same network topology, as seen in Figure 1. The experimental study carried out in this paper included applying VoIP in different MANET scenarios through the network from a random source node to random destinations all over the tested MANET network scenarios. The queues configuration is done on the MANET's nodes since the nodes are working as routers in MANET networks. However the different features of the modeling and simulation experiment can be summarized as follows:

1) Five different wireless ad hoc routing protocols (AODV, OLSR, DSR, TORA, GRP);

2) Each protocol is further analyzed with three different queuing techniques (FIFO, PQ,WFQ);

3) Each protocol is further analyzed also with/without application of queuing;

4) Mobility.

\section{VOIP Traffic Configuration}

VOIP traffic has been configured between nodes by using "create traffic flow" option, with the following input parameters: 


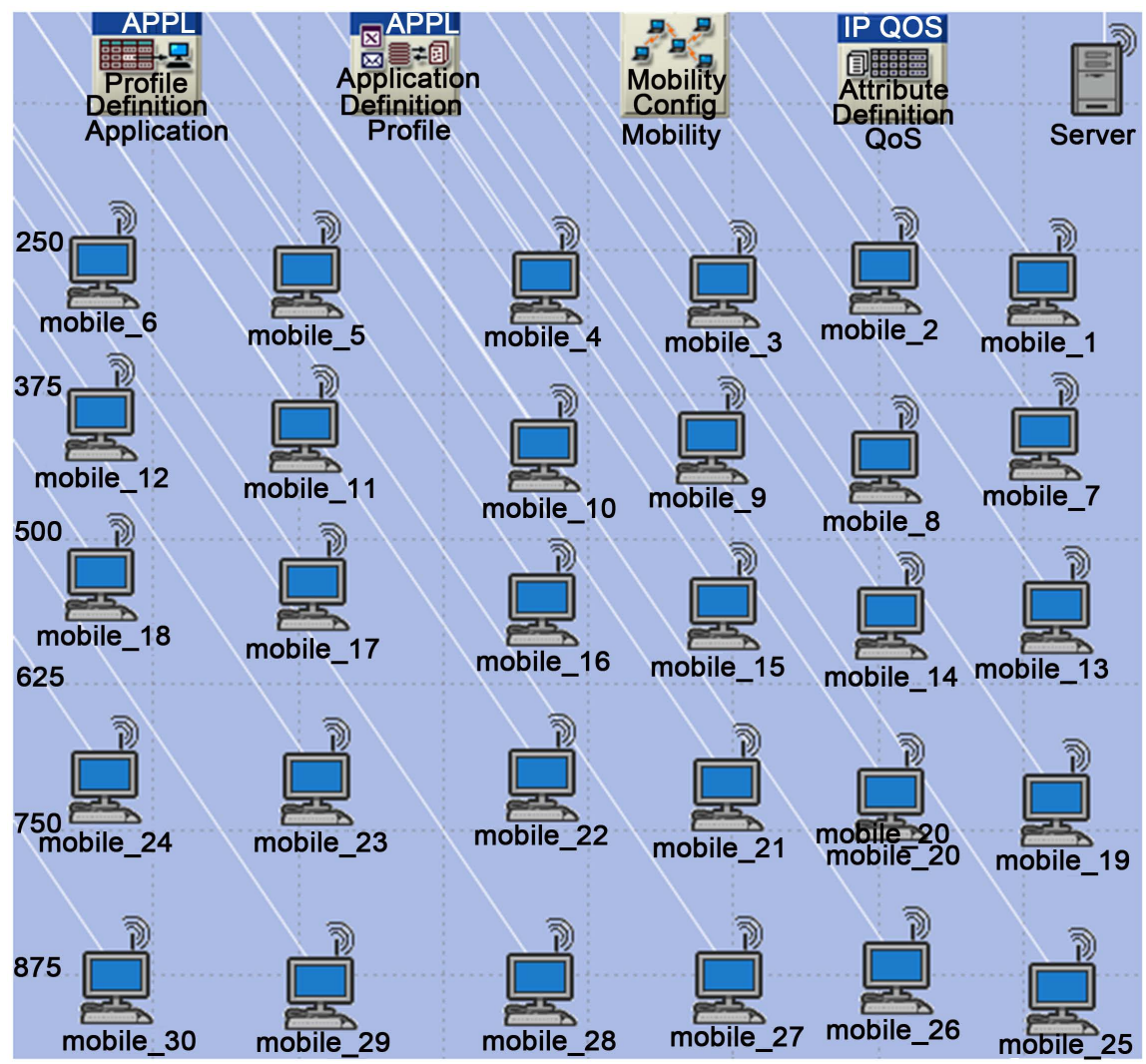

Figure 1. Case study topology with mobile nodes.

- Call rate: 300 calls per hour.

- Traffic generated randomly and concurrently per node and generated from all nodes to all nodes simultaneously. The OPNET produced 870 flows of VoIP traffic for the period of the simulation (1000 seconds) that is equal to a data of 117.294 GB, which indicates the performance analysis; see Figure 2.

- Average call duration: $180 \mathrm{~s}$ (3 min).

- Voice flow duration: $3600 \mathrm{~s} \mathrm{(60} \mathrm{min).}$

- Encoder scheme is G.711.

- Type of service: interactive voice with delay, Throughput, and reliability including overhead (bytes): RTP/UDP/IP.

- 30 MANET nodes.

- The speed of the mobile nodes is configured to be equal to $10 \mathrm{~km} / \mathrm{h}$ with mobility in a curved trajectory in the area of $1 \mathrm{~km} \times 1 \mathrm{~km}$.

- Wireless technology used type: $802.11 \mathrm{~g}$ with $54 \mathrm{Mbps}$ bit rate.

\section{Results and Practical Analysis}

Comparison between wireless ad hoc networks routing protocolsis carried out with the following performance metrics: Jitter (sec), Packet End-to-End delay $(\mathrm{sec})$, Wireless LAN Delay (sec), Load (bit/sec), Throughput (bits/sec). All previous performance metrics were compared in the wireless ad hoc networks with applying routing protocols (AODV, OLSR, TORA, DSR, and GRP) using the following queuing techniques: FIFO, PQ, and WFQ. 


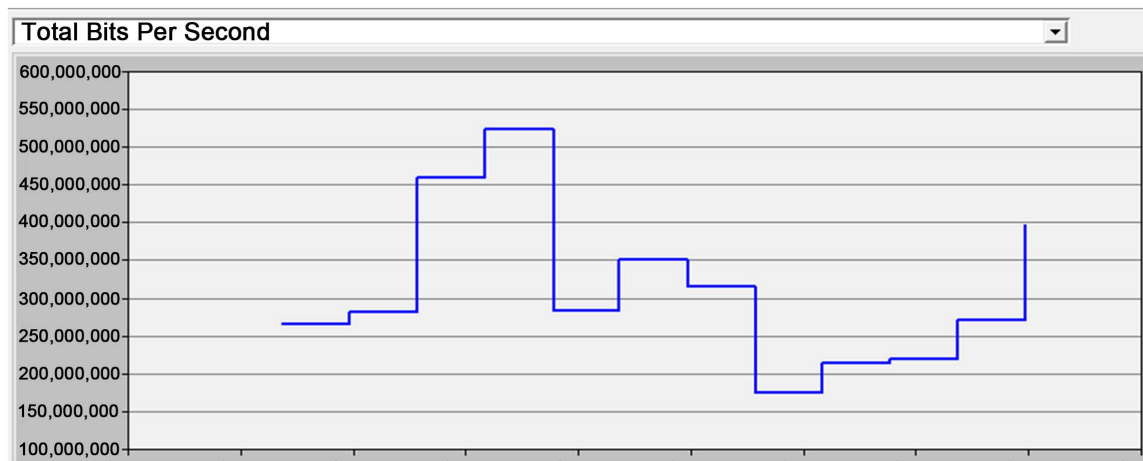

Figure 2. VoIP data injected into the case study (bps).

- In all the following figures, colors are for the routing protocols are: AODV (blue), DSR (Red), GRP (Green), OLSR (Cyan), and TORA (Yellow).

A.Results of the VoIP comparison between AODV, OLSR, TORA, DSR, and GRP using FIFO queuing technique

Figure 3 describes the jitter (sec) for all the targeted routing protocols with FIFO queuing. However, AODV (blue line in the Figure 3) seems to be has recorded the smallest delay jitter value (i.e. near zero value) than other protocols; while the OLSR and DSR have registered the worst delay jitter values, respectively.

Figure 4 illustrates packet end-to-end delay graph results of the candidate MANET protocols with FIFO queuing. The GRP protocol has recorded the highest (worst) value; while TORA protocol has produced the lowest (best) endto-end delay and AODV has granted the second lowest end-to-end delay value after TORA protocol.

Figure 5 shows the wireless LAN media access delay results of the tested protocols with FIFO queuing. It is seen that TORA protocol has got the lowest (best) wireless LAN media access delay with OLSR getting the second least value of the wireless LAN media access delay, while the GRP getting the highest (worst) value of this measurement. Figure 6 refers to the results of the recoded wireless LAN load (bits/sec). In this graph OLSR has reached the highest load value, while the GRP protocol has the lowest (worst) wireless LAN load (bits/ sec) with FIFO queuing.

Figure 7 shows the results of the Throughput comparison with FIFO queuing. The graph states that AODV protocol has gained the highest (best) Throughput value and the OLSR protocol has recorded the second highest value; while GRP has the lowest (worst) Throughput value than other protocols.

By reading Figures 3-7, for VoIP application over the MANET with FIFO queuing, it is clear that in terms of Jitter the AODV is producing the best results while with the end-to-end delay TORA is coming in the first place with best results and AODV is registered the second best results. Moreover the AODV is also gained the highest Throughput, while OLSR produced the second best result of a Throughput after the AODV. Therefore, AODV and OLSR are both protocols are good candidates for VoIP over MANET wireless networks. 


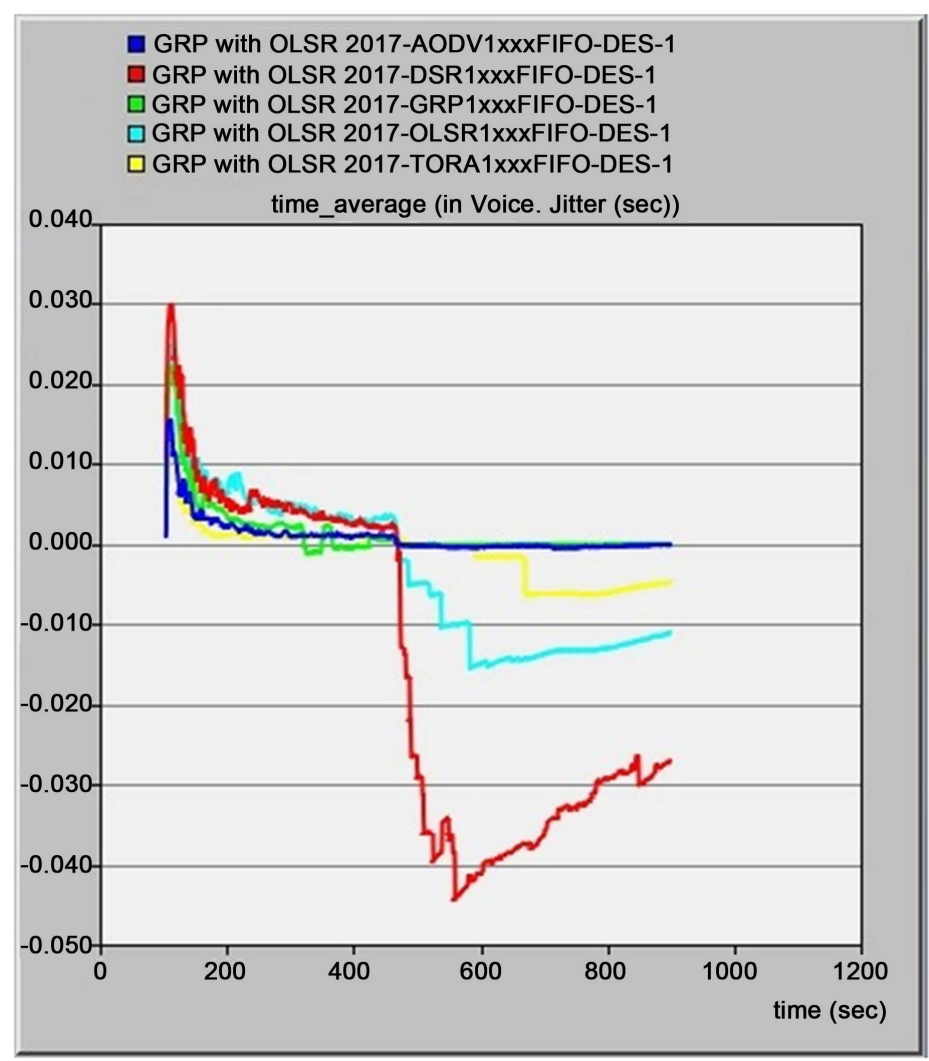

Figure 3. Voice Jitter (sec) results for the routing protocols using FIFO queuing.

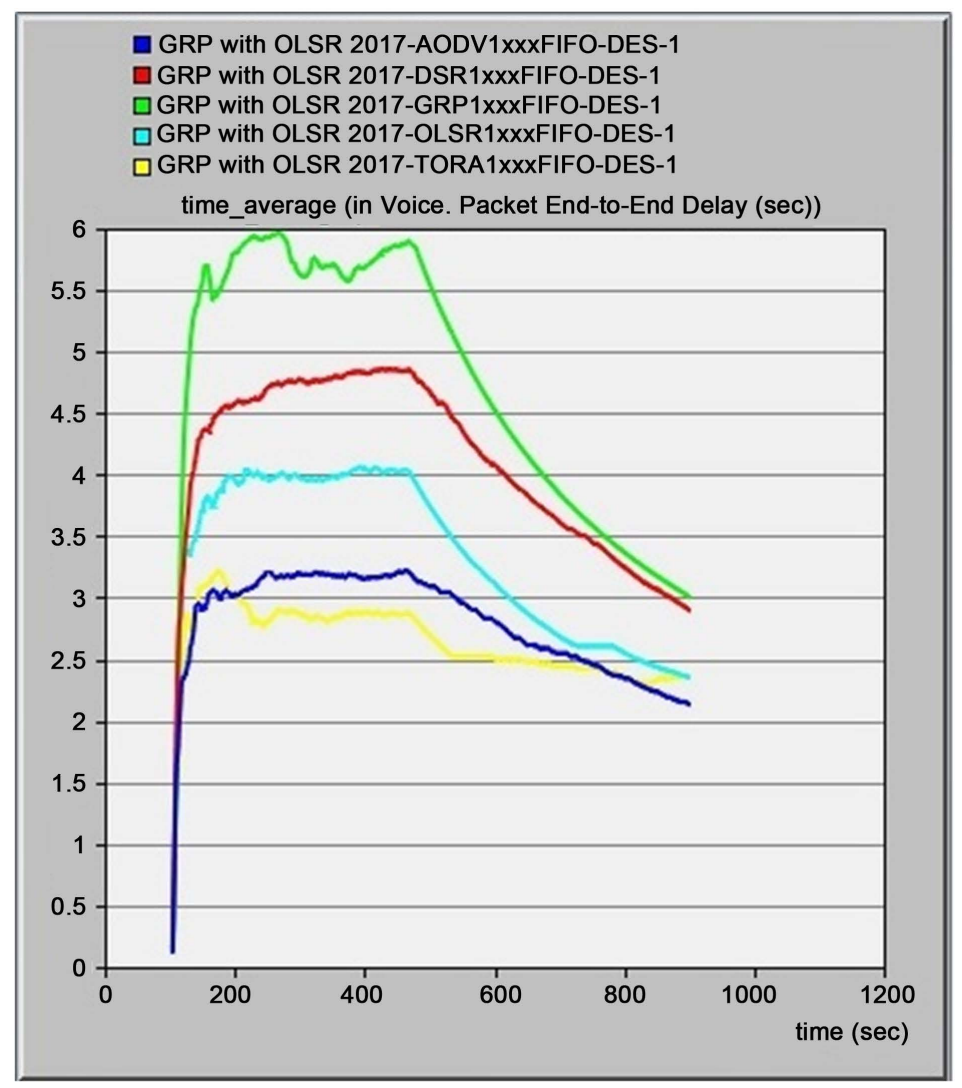

Figure 4. Packet End-to-End Delay (sec) for the routing protocols with FIFO queuing. 


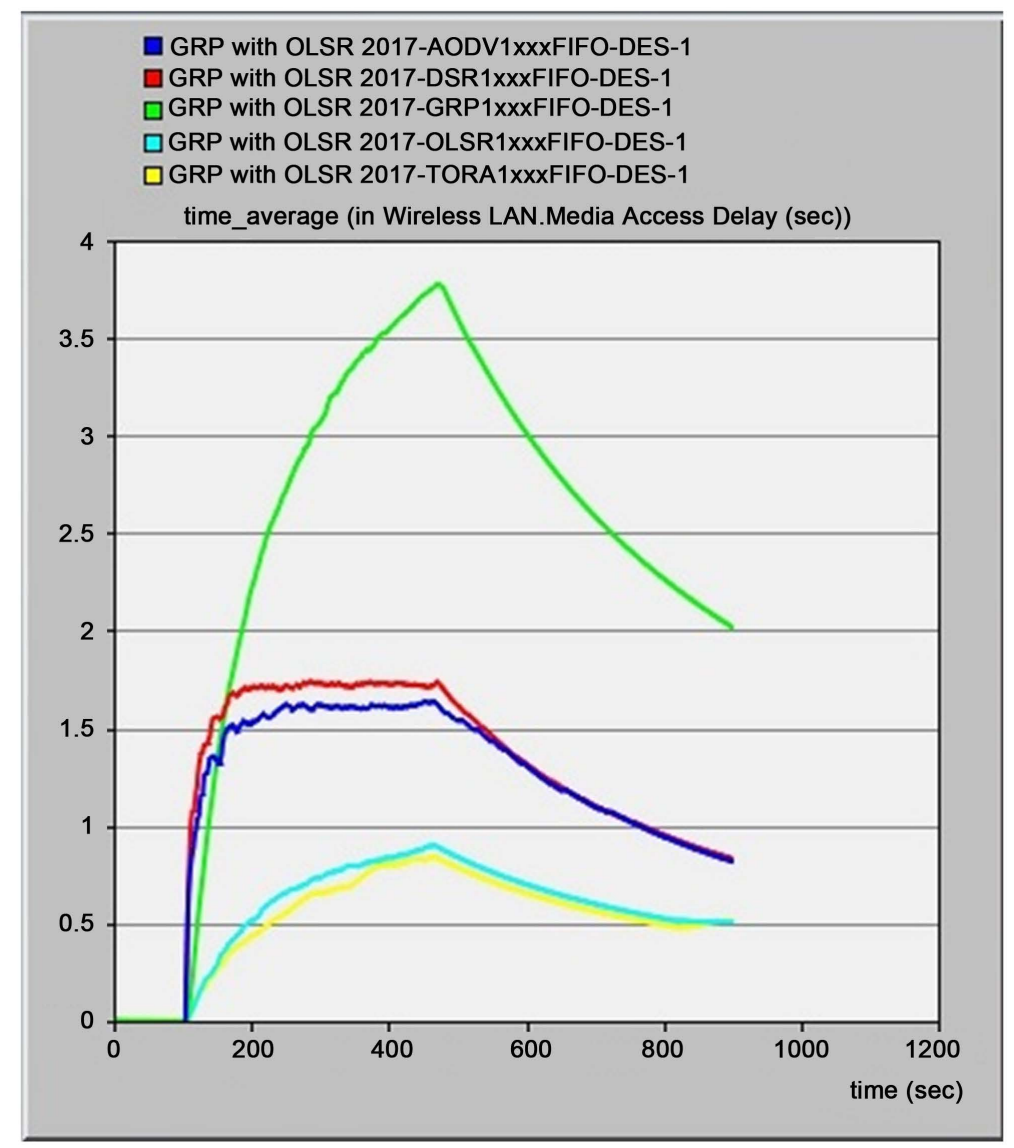

Figure 5. Wireless LAN delay (sec) with FIFO queuing for the routing protocols.

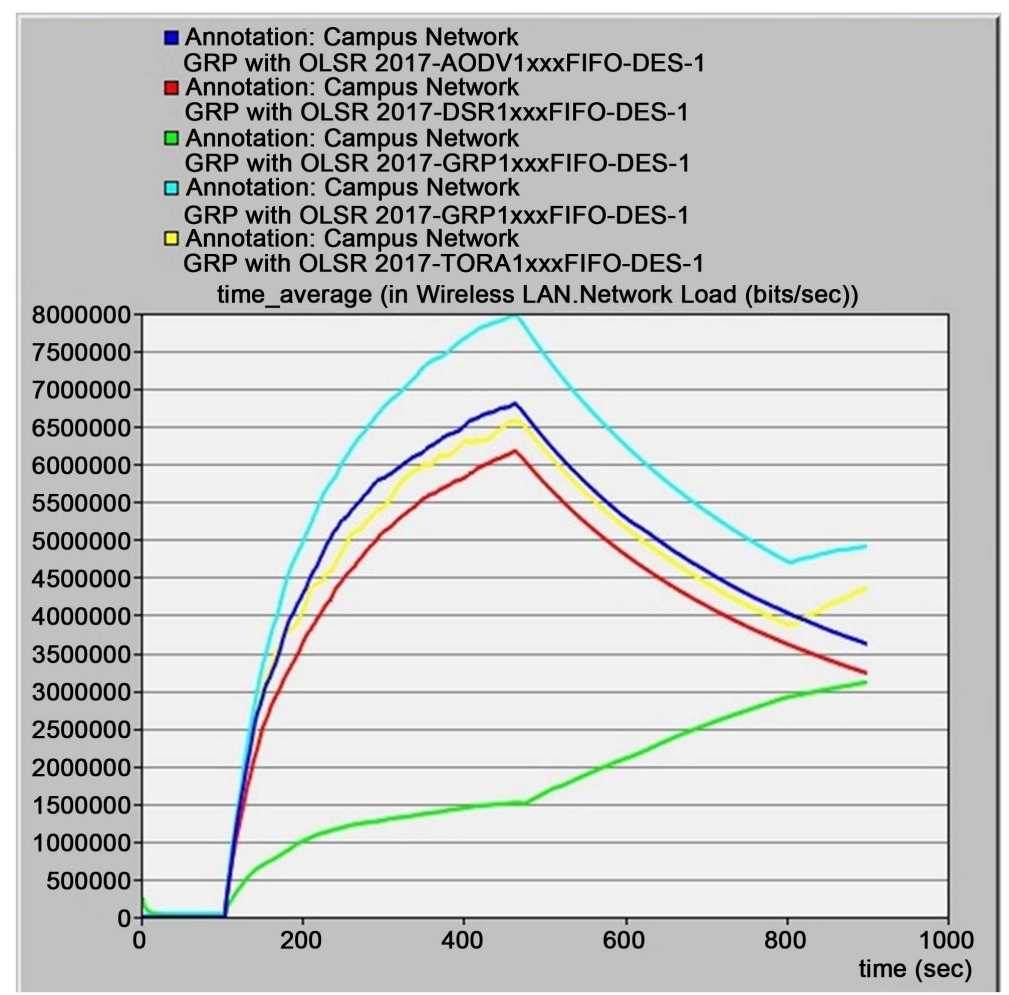

Figure 6. Wireless LAN Load (bit/sec) with FIFO queuing for the routing protocols. 


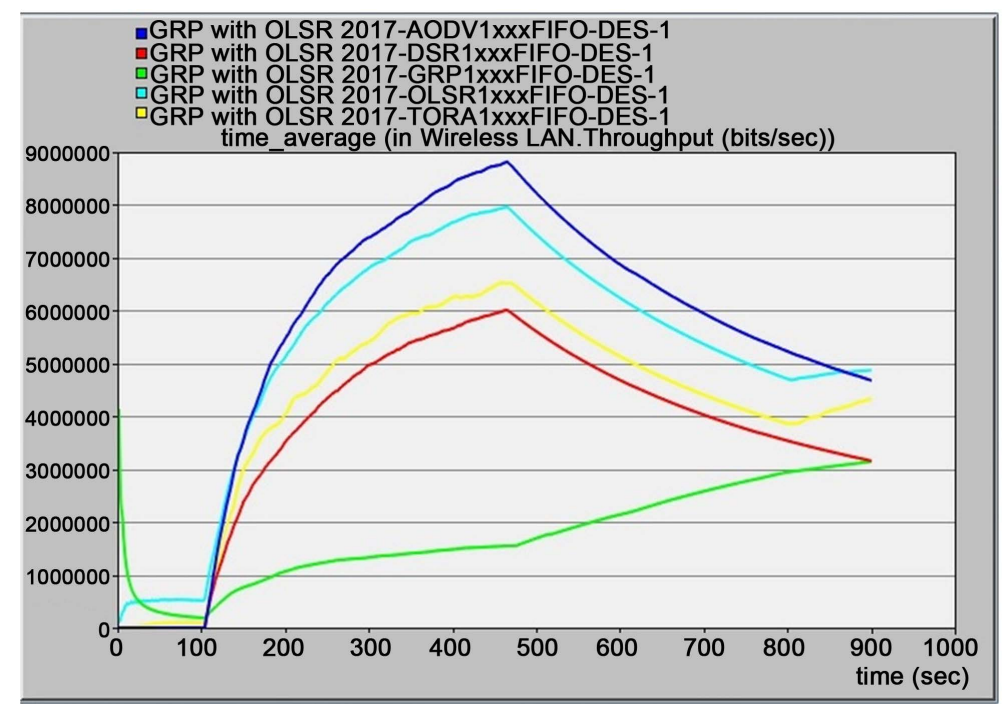

Figure 7. Wireless LAN Throughput (bit/sec) for the routing protocols with FIFO queuing.

\section{B. Results of the VoIP comparison between AODV, OLSR, TORA, DSR, and GRP using $P Q$ queuing technique:}

Figure 8 describes the jitter $(\mathrm{sec})$ for all the targeted routing protocols with PQ queuing. However, AODV (blue line in Figure 8) seems to be has recorded the smallest delay jitter value (i.e. near zero value) than other protocols; while the GRP and DSR have registered the worst delay jitter values, respectively.

Figure 9 illustrates packet end-to-end delay graph results of the candidate MANET protocols with PQ queuing. The GRP protocol has recorded the highest (worst) value; while OLSR protocol has produced the lowest (best) end-to-end delay and AODV has granted the second lowest end-to-end delay value after OLSR protocol.

Figure 10 shows the wireless LAN media access delay results of the tested protocols with PQ queuing. It is seen that TORA protocol has got the lowest (best) wireless LAN media access delay with OLSR getting the second least value of the wireless LAN media access delay, while the GRP receiving the highest (worst) value of this measurement.

Figure 11 refers to the results of the recoded wireless LAN load (bits/sec). In this graph DSR has reached the highest load value, while the TORA protocol has the lowest (worst) wireless LAN load (bits/sec) with PQ queuing.

Figure 12 shows the results of the Throughput comparison with PQ queuing. The graph states that AODV protocol has gained the highest (best) Throughput value and the TORA protocol has recorded the second highest value; while GRP has the lowest (worst) Throughput value than other protocols.

By reading Figures 8-12, for VoIP application over the MANET with PQ queuing, it is clear that in terms of Jitter and end-to-end delays the AODV then OLSR are the two best routing protocols than others. However, in terms of the Throughput the AODV then TORA are gaining the best results. Moreover, TORA protocol has produced the smallest wireless LAN media access delay 


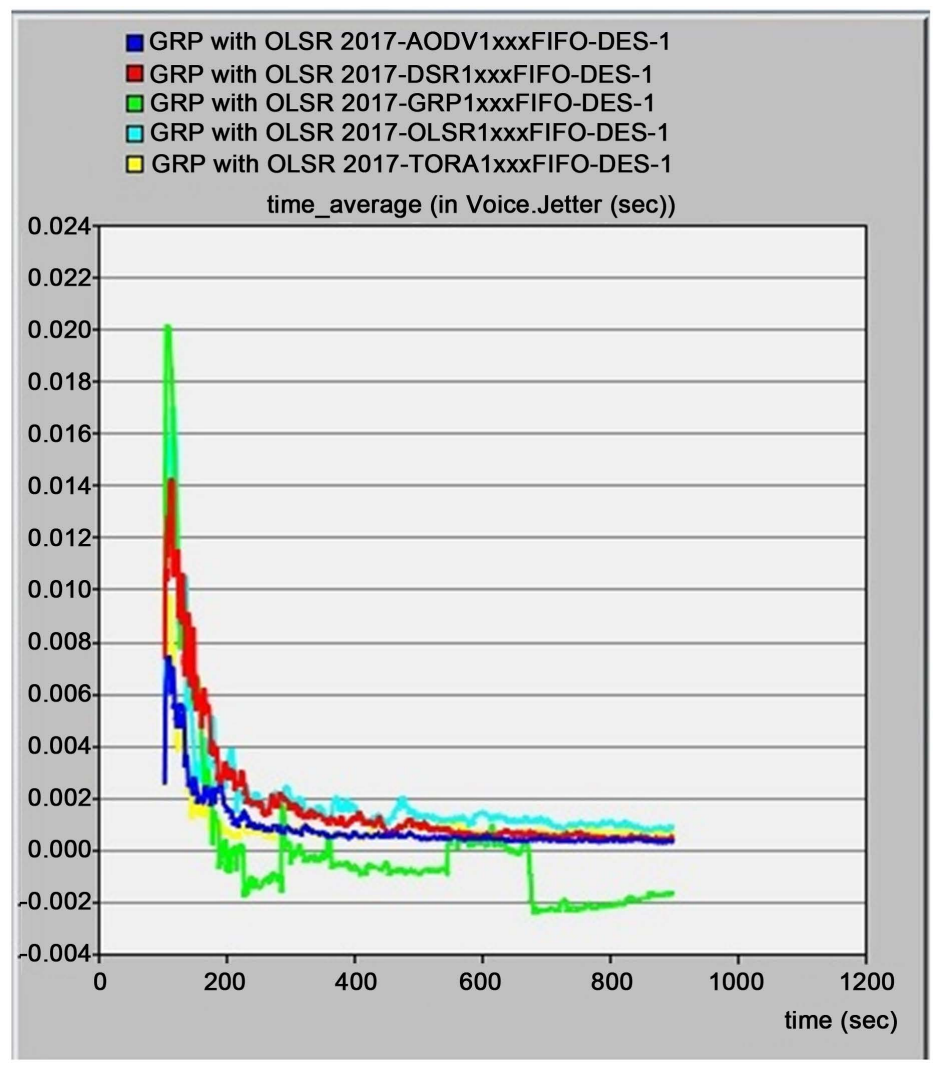

Figure 8. Voice jitter (sec) for the routing protocols using PQ queuing.

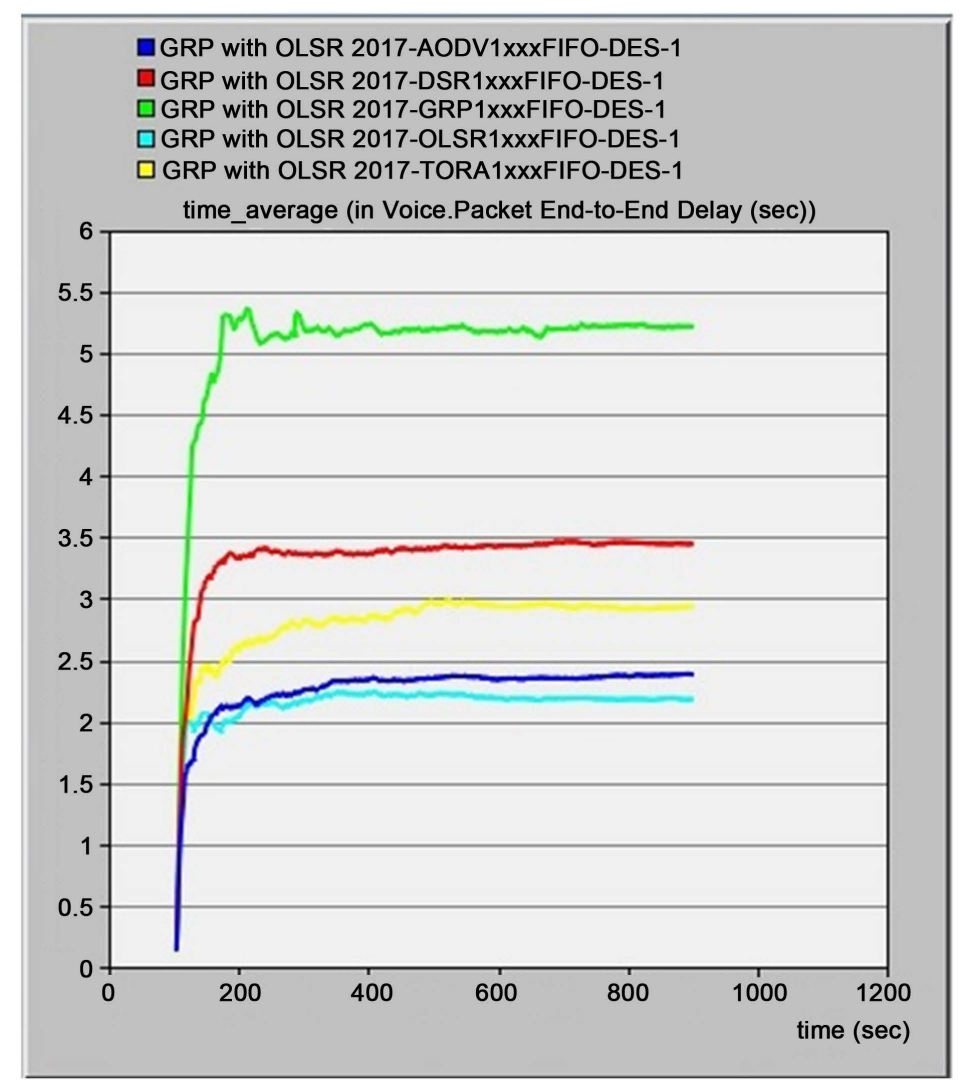

Figure 9. Packet End-to End Delay (sec) using PQ queuing for the routing protocols. 


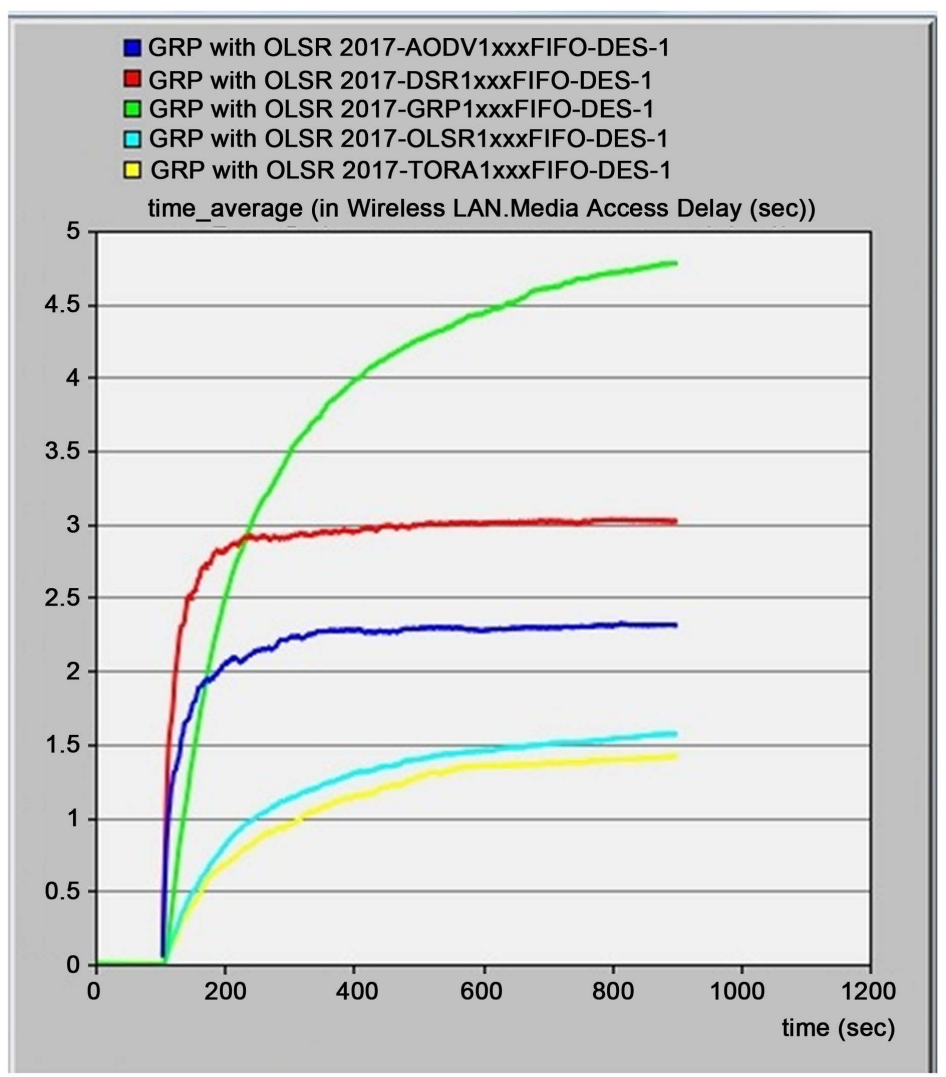

Figure 10. Wireless LAN delay (sec) using PQ queuing for the routing protocols.

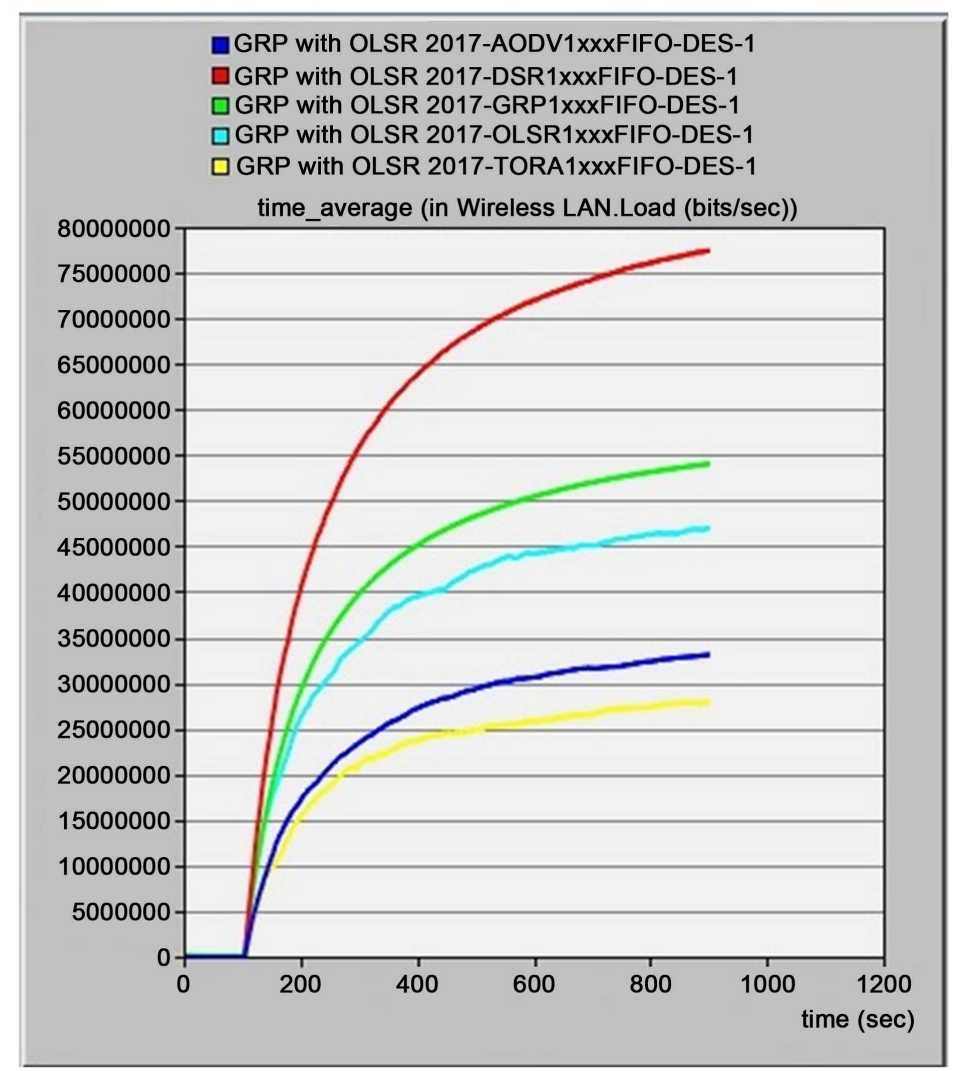

Figure 11. Wireless LAN Load (bit/sec) using PQ queuing for the routing protocols. 


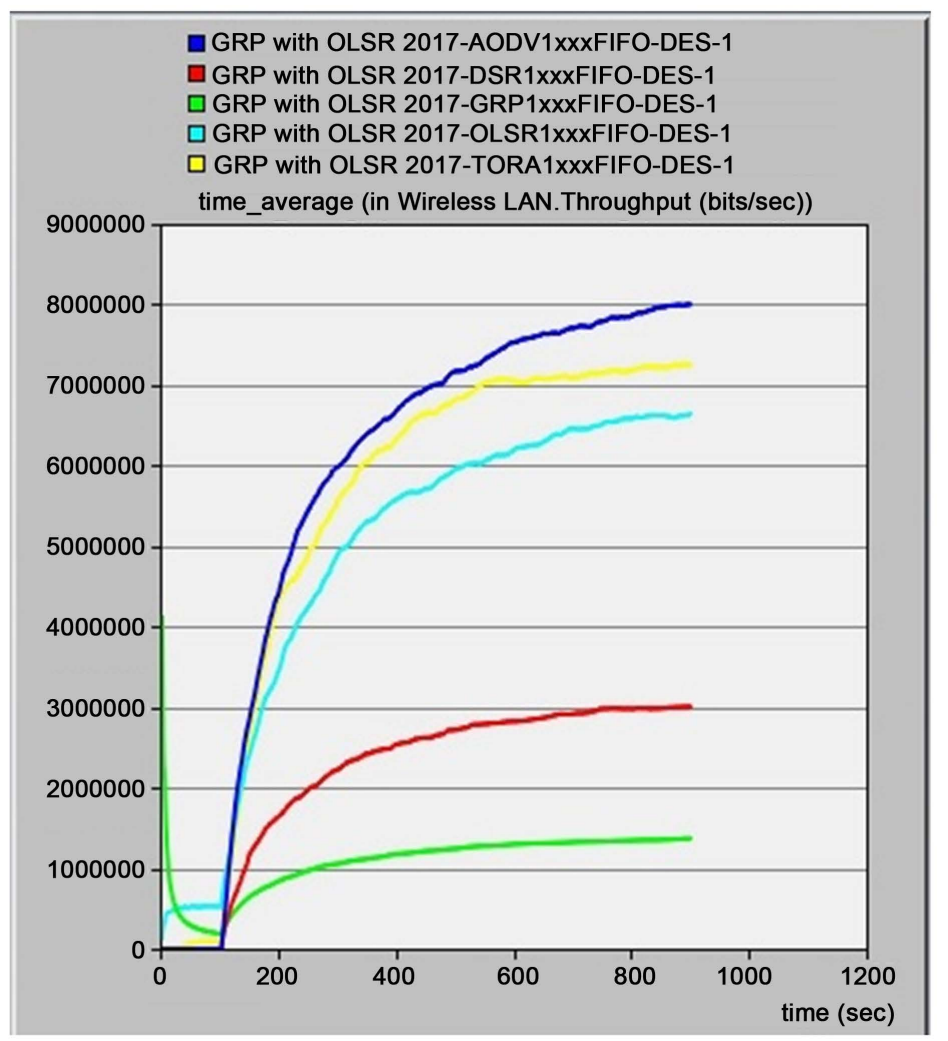

Figure 12. Wireless LAN Throughput (bit/sec) using PQ queuing for the routing protocol.

while OLSR is came in the second place. Consequently, AODV and OLSR are both protocols are good candidates for VoIP over MANET wireless networks.

\section{Results of the VoIP comparison between AODV, OLSR, TORA, DSR, and GRP using WFQ queuing technique:}

Figure 13 describes the jitter (sec) for all the targeted routing protocols with WFQ queuing. However, AODV (blue line in Figure 13) seems to be has recorded the smallest delay jitter value (i.e. near zero value) than other protocols; while the OLSR and GRP have registered the worst delay jitter values, respectively.

Figure 14 illustrates packet end-to-end delay graph results of the candidate MANET protocols with WFQ queuing. The GRP protocol has recorded the highest (worst) value; while AODV protocol has produced the lowest (best) endto-end delay and OLSR has granted the second lowest end-to-end delay value after OLSR protocol.

Figure 15 shows the wireless LAN media access delay results of the tested protocols with WFQ queuing. It is seen that TORA protocol has got the lowest (best) wireless LAN media access delay with OLSR getting the second least value of the wireless LAN media access delay, while the GRP receiving the highest (worst) value of this measurement.

Figure 16 refers to the results of the recoded wireless LAN load (bits/sec). In this graph GRP has reached the highest load value, while the TORA protocol has the lowest (worst) wireless LAN load (bits/sec) with WFQ queuing. 


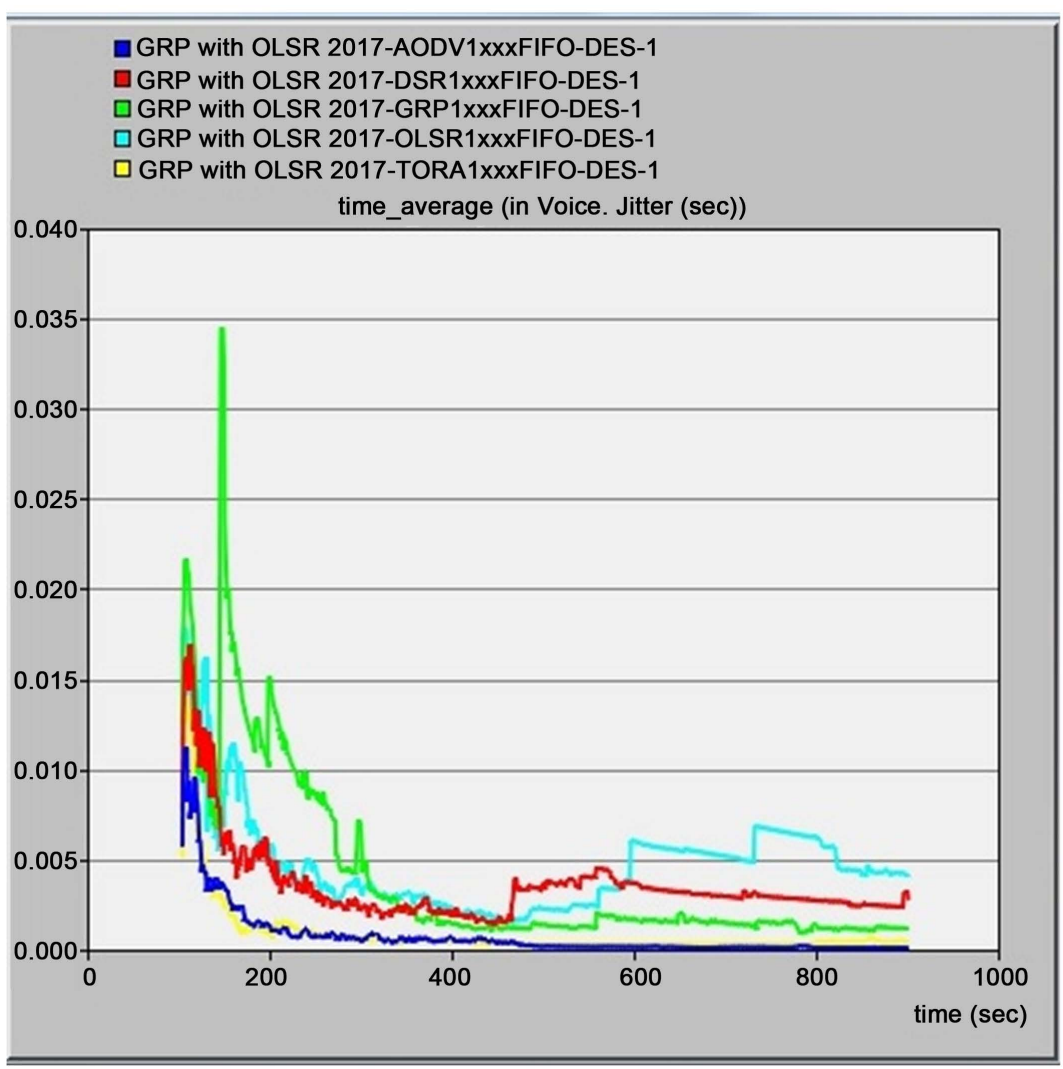

Figure 13. Jitter (sec) using WFQ queuing for the routing protocol.

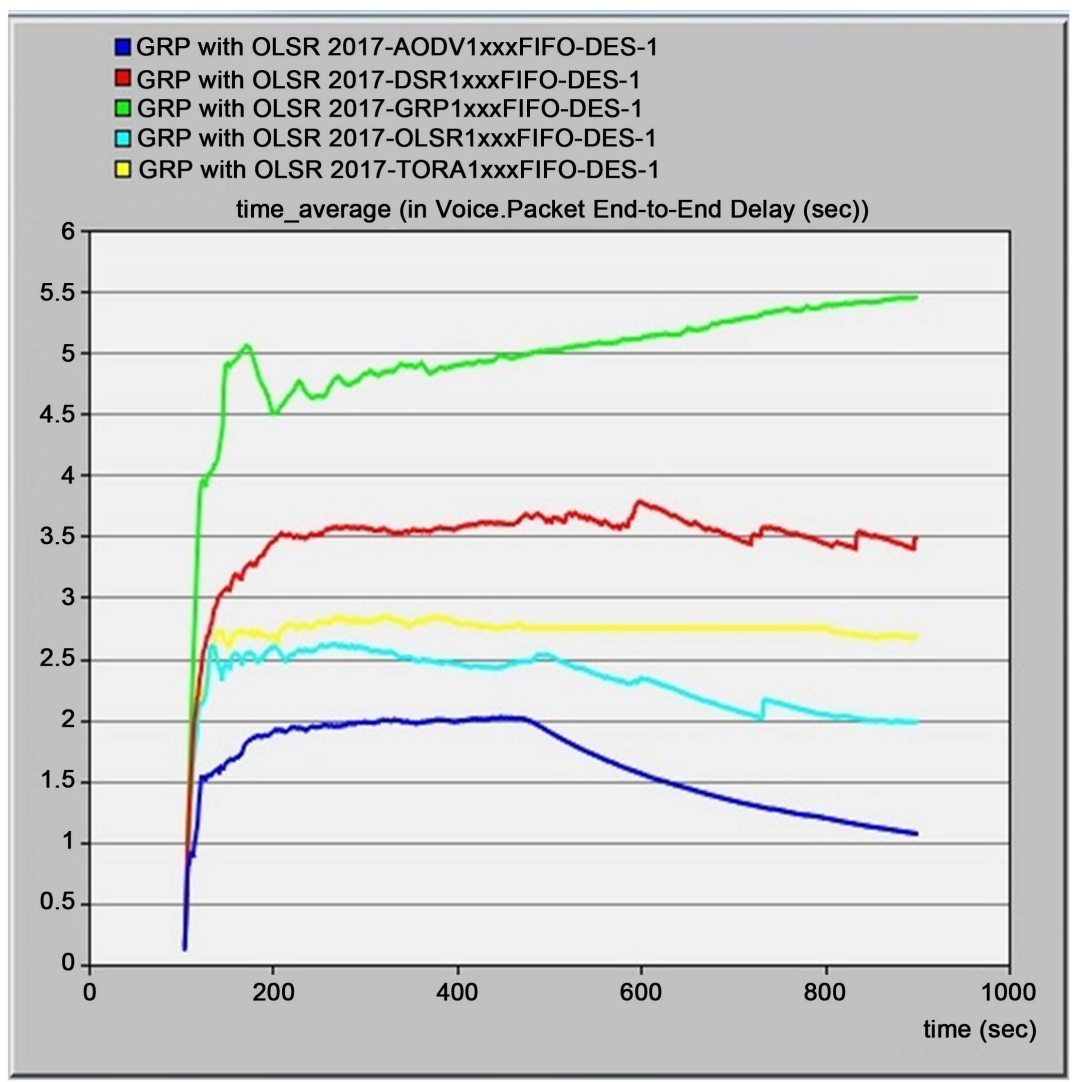

Figure 14. Packet End-to End delay (sec) using WFQ queuing for the routing protocol. 


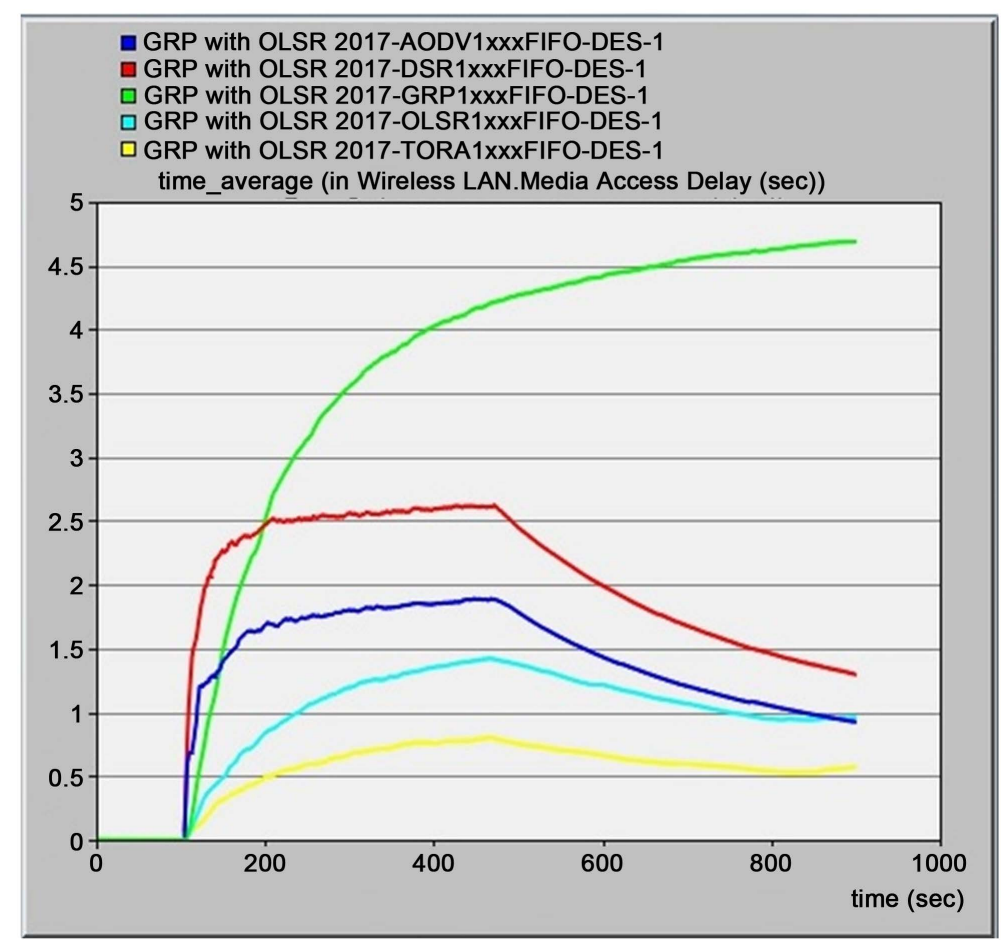

Figure 15. Wireless LAN delay (sec) using WFQ queuing for the routing protocol.

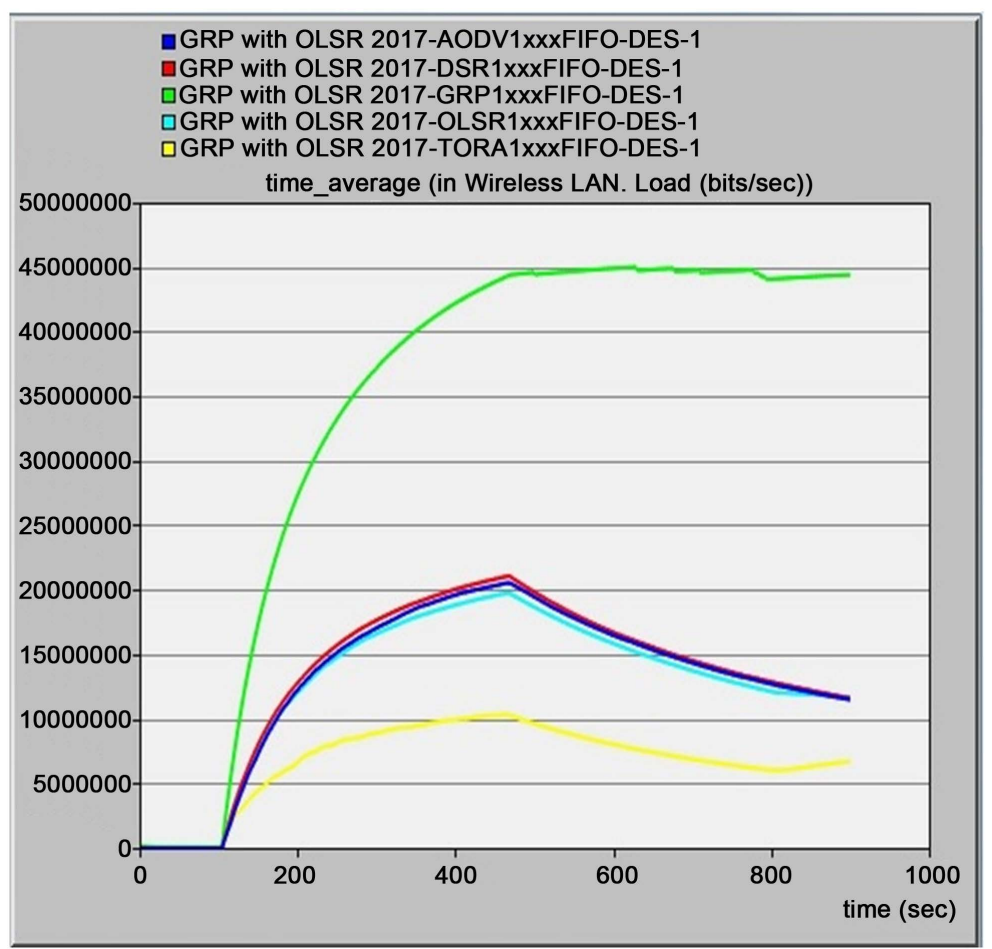

Figure 16. Wireless LAN Load (bit/sec) using WFQ queuing for the routing protocol.

Figure 17 shows the results of the Throughput comparison with WFQ queuing. The graph states that AODV protocol has gained the highest (best) Throughput value and the TORA protocol has recorded the second highest value; while GRP has the lowest (worst) Throughput value than other protocols. 


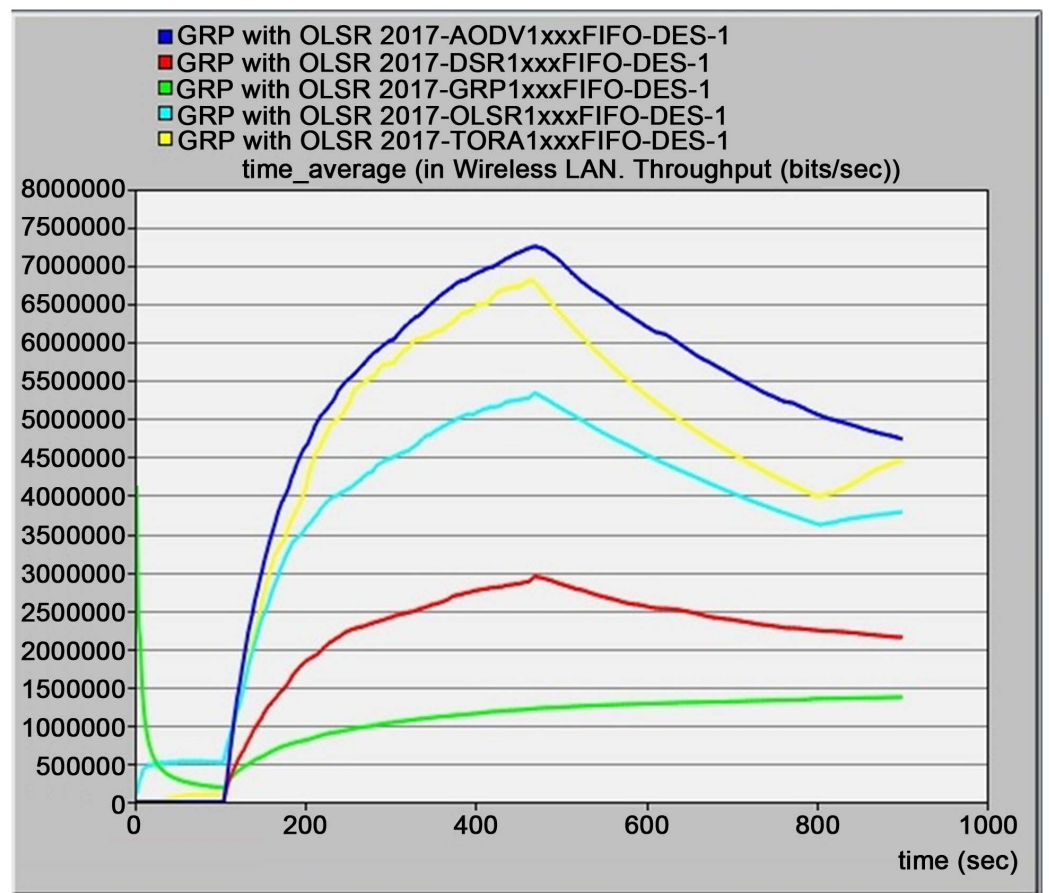

Figure 17. Wireless LAN throughput (bit/sec) using WFQ queuing for the routing protocol.

By reading Figures 13-17, for VoIP application over the MANET with WFQ queuing, it is clear that in terms of Jitter and end-to-end delay and the Throughput that the AODV is the best candidate among the experimented routing protocols.

\section{Conclusions}

This paper presents an analysis study about running VoIP over the MANET networks with different routing protocols using different queuing mechanisms with OPNET tool. However, in this case study, in terms of voice delay jitter, the AODV produced good results than other tested protocols in all presented queuing mechanisms. In terms of end-to-end delay, the TORA recorded the best result with FIFO queuing, while OLSR has got the best result with PQ queuing, and AODV has got good results with WFQ case. Moreover, TORA protocol has showed good results in terms of wireless media delay measurement with all the three types of queuing. In another hand, AODV protocol has presented good performance measures for the throughput values with different queuing techniques.

The final conclusion is as following: the AODV and OLSR are the two best protocols that can be used with VoIP application over the MANET wireless networks, which are coming due to routing mechanisms employed in these two protocols.

\section{References}

[1] Fan, K.-W., Liu, S. and Sinha, P. (2006) Ad Hoc Routing Protocols. In: Boukerche, 
A., Ed., Handbook of Algorithms for Wireless Networking and Mobile Computing, Chapman \& Hall/CRC Publisher, Boca Raton.

[2] Hanzo, L. II and Tafazolli, R. (2007) A Survey of QOS Routing Solutions for Mobile Ad Hoc Networks. IEEE Communications Surveys \& Tutorials, 9, 50-70.

[3] Jain, R. (1991) The Art of Computer Systems Performance Analysis: Techniques for Experimental Design, Measurement, Simulation, and Modeling. Wiley Publisher, Hoboken.

[4] Kurose, J.F. and Ross, K.W. (2012) Computer Networking: A Top-Down Approach Featuring the Internet. Pearson, Upper Saddle River.

[5] Sllame, A.M. and Aljafry, M. (2013) Using Simulation and Modeling Tools in Teaching Computer Network Courses. IEEE International Conference on IT Convergence and Security, Macau, 16-18 December 2013, 1-4.

[6] OPNET Technologies Inc. (2004) OPNET Product Documentation v.11.0.A. OPNET Technologies, Inc., Bethesda.

[7] Audah, L., et al. (2015) Performance Evaluation of Voice over IP Using Multiple Audio Codec Schemes, in Asian Research Publishing Network (ARPN). Journal of Engineering and Applied Sciences, 10, 8912-8919.

[8] Ravikanti, S. and Preeti, G. (2015) Evaluating the Performance of Reactive Unicast Routing Protocols with OPNET Simulator in MANETS under VOIP. International Journal of Innovative Research in Science, Engineering and Technology, 4, 57015710 .

[9] Jasani, H. (2012) Evaluations of AODV and DSR for QoS Requirements. ACM SIGITE 12 Conference, Calgary, 11-13 October 2012.

[10] Sanchez-Iborra, R. and Cano, M.-D. (2014) An Approach to a Cross Layer-Based QoE Improvement for MANET Routing Protocols. Network Protocols and Algorithms Journal, 6, 18-34.

[11] Cai, L., et al. (2006) VoIP over WLAN: Voice Capacity, Admission Control, QoS, and MAC. International Journal of Communication Systems, 19, 491-508. https://doi.org/10.1002/dac.801

[12] Vijayakumar, M. and Karthikeyani, V. (2016) An Enhanced Perceptive Queuing Technique (CBCRTQ) for Traffic Management in VoIP over MANET. International Journal of Future Generation Communication and Networking, 9, 99-112.

[13] Mohammed, S.O. and Nabi Mustafa, A.B. (2016) VoIP over MANET Routing Protocols OLSR \& TORA Performance Evaluation. International Journal of Science and Research, 5, 819-823.

[14] Di Caro, G.A., Ducatelle, F. and Gambardella, L.M. (2008) A Simulation Study of Routing Performance in Realistic Urban Scenarios for MANETs. Proceedings of ANTS 2008, 6th International Workshop on Ant Algorithms and Swarm Intelligence, Brussels, 22-24 September 2008, LNCS 5217.

[15] Mittal, K. and Sharma, P. (2015) Performance Evaluation of MANET Routing Protocols for VOIP Applications. International Journal of Science, Engineering and Technology Research, 4, 1977-1981.

[16] El brak, S., Bouhorma, M. and Boudhir, A.A. (2011) VoIP over MANET (VoMAN): QoS \& Performance Analysis of Routing Protocols for Different Audio Codecs. International Journal of Computer Applications, 36, 22-26.

[17] Sllame, A.M., et al. (2015) Performance Comparison of VoIP over Wireless Ad Hoc Networks Using Different Routing Protocols and Queuing Techniques. IEEE International Symposium on Networks, Computers and Communications, Hammamet, 
13-15 May 2015, 1-6.

[18] Evans, J. and Filsfils, C. (2007) Deploying IP and MPLS QOS for Multiservice Networks: Theory and Practice. Morgan Kaufmann Publishers, Burlington.

Open Access Library

Submit or recommend next manuscript to OALib Journal and we will provide best service for you:

- Publication frequency: Monthly

- 9 subject areas of science, technology and medicine

- Fair and rigorous peer-review system

- Fast publication process

- Article promotion in various social networking sites (LinkedIn, Facebook, Twitter, etc.)

- Maximum dissemination of your research work

Submit Your Paper Online: Click Here to Submit

Or Contact service@oalib.com 